\title{
Editorial Bildungsungleichheit und Gerechtigkeit in der Schweiz
}

\section{Rolf Becker}

Soziale Ungleichheit von Bildungschancen und Fragen der Gerechtigkeit beim Zugang zu und Erwerb von Bildung gehören - nach den bereits lange zurückliegenden Debatten in den 1960er Jahren - auch in der Schweiz wieder zu den zentralen Forschungsgebieten der Bildungswissenschaft (vgl. Becker, 2010; Buchmann et al. 2007; Buchmann \& Charles, 1993; Hadjar \& Berger, 2010; Jann \& Combet, 2012). Dass in der Schweiz wie in allen anderen modernen Gesellschaften trotz Bildungsexpansion und vieler Bildungsreformen weiterhin soziale Ungleichheiten von Bildungschancen nach sozialer Herkunft, Migrationshintergrund, Geschlecht und Region bestehen, ist nicht nur häufig empirisch belegt worden, sondern zählt mittlerweile auch zum Alltagswissen. Wie in vielen anderen europäischen Ländern (vgl. Breen et al., 2009, 2010, 2012), hat sich auch in der Schweiz im Zuge der Bildungsexpansion die herkunftsbedingte Bildungsungleichheit verringert (vgl. Hadjar \& Berger, 2010). Während im Jahre 1960 rund 4 Prozent der 19-Jährigen (in der ständigen Wohnbevölkerung) die gymnasiale Maturität und damit die Berechtigung für ein Universitätsstudium erworben hatten, lag die Quote der gymnasialen Maturität im Jahre 1970 bei 7 und 1980 bei rund 10 Prozent. Im Jahre 2010 konnte rund ein Fünftel der 19-Jährigen die Schulbildung mit der gymnasialen Maturität abschliessen. Wird die Berufsmaturitätsquote hinzugerechnet, so hatte im Jahre 2010 rund ein Drittel der altersgleichen Bevölkerung die Hochschulberechtigung erworben. Seit Anfang der 1990er Jahre haben die jungen Frauen die altersgleichen Männer beim Erwerb der Maturität und mit zeitlicher Verzögerung auch beim Hochschulzugang überflügelt. Dennoch hängt der Erwerb höherer Bildung immer noch sehr stark von der sozialen Herkunft ab, so dass hierbei Kinder mit sozial privilegierter Herkunft im Vorteil sind (Becker \& Zangger, 2013; Buchmann et al., 2007; Jann \& Combet, 2012).

In der jüngsten Gegenwart konnten die Mädchen und Frauen ihre Bildungsrückstände gegenüber den Knaben und Männern mehr als wettmachen. So drehte sich bis 2010 das Geschlechterverhältnis beim Erwerb der Maturität um (vgl. Becker et al., 2013). Bei der gymnasialen Maturität setzte die Umkehr der 
Geschlechterrelationen bereits früher zu Anfang der 1990er Jahre ein. Auch bei der Berufsmaturität haben die Frauen ihren Rückstand gegenüber den Männern inzwischen egalisiert. Derzeit verfügen rund 35 Prozent der Frauen und rund 30 Prozent der Männer über eine Hochschulberechtigung. Beim Studien- und Berufsabschluss haben die Frauen ebenfalls die altersgleichen Männer überflügelt. Während die Knaben eher die Schule ohne Abschluss verlassen, haben Frauen im Bereich der Berufsbildung vergleichsweise Nachteile gegenüber den jungen Männern.

Des Weiteren sind im Schweizer Bildungssystem die Kinder und Jugendlichen mit Migrationshintergrund gegenüber den Einheimischen im Nachteil. In allen Bereichen des Bildungssystems haben sie geringere Chancen, für den Bildungserfolg günstige Leistungen zu erzielen, erfolgreich ihre Schul- und Berufsausbildung abzuschliessen und die anvisierten Bildungserträge auf dem Arbeitsmarkt zu erzielen. Für Migrantenkinder aus ressourcenarmen Elternhäusern besteht ebenso wie für einheimische Kinder aus den unteren Sozialschichten ein hohes Risiko für Ausbildungslosigkeit sowie für Kompetenz- und Bildungsarmut. In der Zwischenzeit konnte auch für die Schweiz nachgewiesen werden, dass die Nachteile von Kindern und Jugendlichen im Schul- und Hochschulsystem im Wesentlichen ein Spezialfall des Zusammenhangs von sozialer Herkunft und Bildungschancen sind (vgl. Beck et al., 2010; Becker et al., 2013). In dieser Hinsicht stellt die Schweiz keine Ausnahme dar: Zwar erbrachte die Bildungsexpansion, die in der Schweiz vornehmlich aus ökonomischen Gründen vorangebracht wurde, einen Zuwachs an Bildungschancen für alle Sozialgruppen, aber keinen umfassenden Abbau der sozialen Ungleichheit von Bildungschancen.

Während die Ungleichheit der Bildungsergebnisse (d. h. Verteilung von Bildungsabschlüssen) zwischen sozialen Gruppen und Regionen kaum als ungerecht oder ungerechtfertigt angesehen wird, da sie zumeist mit nachweislichen Unterschieden in den Schulleistungen einhergeht, wird Chancenungleichheit im Bildungssystem als illegitim und ungerecht angesehen. «Auch das Gebot der Chancengleichheit gilt nicht uneingeschränkt, sondern wiederum nur insoweit, als nicht allgemein annehmbare Gründe eine ungleiche Verteilung von Chancen rechtfertigen" (Ditton, 2004, S. 251). So gelten Talent und Anstrengung, die über die Vergabe von Schulnoten und Abschlusszeugnisse zertifiziert werden, als legitime Gründe für ungleiche Bildungschancen. Allerdings übersieht diese meritokratische Sichtweise, dass Begabungen sozial ungleich verteilt sind, und Leistungsbereitschaften ebenfalls durch leistungsfremde Kriterien - wie etwa die Klassenlage oder die sozioökonomischen Ressourcen des Elternhauses als Indikatoren für die soziale Herkunft der nachwachsenden Generation - ungleich verteilt werden. So führen ungleiche Ausgangsbedingungen in einem scheinbar fairen Wettbewerb im Bildungssystem zu einer Reproduktion bereits bestehender sozialer Ungleichheiten. Der Wettbewerb ist eben scheinbar fair, weil in der Regel kein Chancenausgleich nach der Einschulung in die Primarstufe erfolgt, 
und durch die Gleichbehandlung ungleicher Personen wird die sozial ungleiche Ausgangslage bei den Lernvoraussetzungen konserviert. Dass eine vorschulische Bildung und Erziehung alleine nicht ausreicht, dieses Problem zu beheben, belegt eine Vielzahl verschiedener Studien. Weil trotz vorheriger Teilhabe an vorschulischer Bildung die sozial differenziellen Einflüsse des Elternhauses auch nach der Einschulung wirken, verblasst in zunehmendem Mass diese Form des Chancenausgleichs.

Dass die meritokratischen Prinzipien problematisch sind, lässt sich am empirischen Beispiel für den Übertritt von der Primarstufe in die Schullaufbahnen der Sekundarstufe I demonstrieren. Herangezogen werden Daten für den Kanton Zürich in den Jahren 1998 (Moser \& Rhyn, 1999, 2000) und 2008 (Moser et al., 2011). Während im Jahre 1998 rund 64 Prozent der Schulkinder am Ende der Primarschulzeit auf die Sekundarschule oder auf das Langzeitgymnasium und 36 Prozent auf die Ober- oder Realschule gewechselt haben, lag die Übergangsrate für die höheren Schullaufbahnen (Sekundarschule Abteilung A und Gymnasium) im Vergleich zum Übergang in die Abteilungen B und C in der Sekundarschule (vergleichbar mit der früheren Ober- und Realschule) rund 10 Jahre später im Jahre 2009 bei 61 versus 39 Prozent. Die soziale Ungleichheit von Bildungschancen ist geringfügig zurückgegangen. Während im Jahre 1998 die Akademikerkinder eine $3 \mathrm{Mal}$ bessere Chance als die Kinder aus den anderen Sozialschichten (Übertrittrate von 82 vs. 60 \%) hatten, auf die höheren Schullaufbahnen zu wechseln, sank dieses Chancenverhältnis auf 2,3 (Übertrittsrate von 75 vs. $57 \%$ ) im Jahre 2009, so dass Akademikerkinder immer noch eine 2 Mal höhere Chance haben, auf das Gymnasium zu gelangen.

Tabelle 1: Reduktion der Bildungsungleichheit durch Neutralisierung der sozialen
Herkunft beim Übergang von der Primar-auf die Sekundarstufe I (Zürich 1998)

\begin{tabular}{rccc}
\hline & \multicolumn{3}{c}{ Kinder aus Akademikerfamilien } \\
\cline { 2 - 4 } haben Notendurchschnitt in Deutsch: & (sehr) gut & befriedigend & mangelhaft \\
\cline { 2 - 4 } und wechseln auf die höheren Schullaufbahnen & $99.3 \%$ & $30.6 \%$ & $7.8 \%$ \\
\cline { 2 - 4 } & \multicolumn{3}{c}{ Kinder aus anderer Sozialschicht } \\
\cline { 2 - 4 } haben Notendurchschnitt in Deutsch: & (sehr) gut & befriedigend & mangelhaft \\
\cline { 2 - 4 } und wechseln auf die höheren Schullaufbahnen & $98.2 \%$ & $52.1 \%$ & $13.9 \%$ \\
\hline
\end{tabular}

Auch die sozial ungleichen Leistungsverteilungen und Übertritte in die höheren Sekundarschulen haben sich wenig geändert. Zum einen sind die Zusammenhänge zwischen sozialer Herkunft (Vater mit Maturität oder Hochschulabschluss vs. andere Bildungsgruppen) und den Schulnoten in Deutsch als Schulfach - also die primären Herkunftseffekte nach Boudon (1974) - offensichtlich. Im Jahre 1998 wiesen rund 62 Prozent der Kinder von Eltern mit höherer Bildung 
gute bis sehr gute Deutschnoten auf, aber nur 34 Prozent der Kinder von Eltern mit einer anderen Bildung (Tabelle 1). Im Jahre 2009 hat sich die herkunftsbedingte Verteilung von Schulleistungen (in Deutsch) nicht gravierend geändert (Tabelle 2).

Tabelle 2: Reduktion der Bildungsungleichheit durch Neutralisierung der sozialen Herkunft beim Übergang von der Primar-auf die Sekundarstufe I (Zürich 2009)

\begin{tabular}{rccc}
\hline \hline & \multicolumn{3}{c}{ Kinder aus Akademikerfamilien } \\
\cline { 2 - 4 } haben Notendurchschnitt in Deutsch: & $54.9 \%$ & $37.4 \%$ & $7.7 \%$ \\
und wechseln auf die höheren Schullaufbahnen & $99.1 \%$ & $53.3 \%$ & $6.5 \%$ \\
\hline & \multicolumn{3}{c}{ Kinder aus anderer Sozialschicht } \\
\cline { 2 - 4 } haben Notendurchschnitt in Deutsch: & (sehr) gut & befriedigend & mangelhaft \\
und wechseln auf die höheren Schullaufbahnen & $96.2 \%$ & $45.9 \%$ & $15.8 \%$ \\
\hline
\end{tabular}

Offenkundig ist aber, dass die Übertrittsraten bei gleicher Schulleistung zwischen den beiden verglichenen Sozialschichten deutlich unterschiedlich ist. Es liegen offensichtlich sekundäre Effekte der sozialen Herkunft vor. Zwar wechseln Kinder aus Akademikerfamilien zu 99,3 Prozent und Kinder aus den anderen Sozialschichten zu 98,2 Prozent auf die höheren Schullaufbahnen, wenn sie gute bis sehr gute Noten aufweisen. Auf den ersten Blick scheint das mit der meritokratischen Leistungsideologie übereinzustimmen. Aber für die anderen Leistungskategorien sind eben deutliche Unterschiede festzustellen. Bei mittelmässigen Leistungen in Deutsch wechseln zwei Drittel der Akademikerkinder, aber nur die Hälfte der anderen Kinder auf die höheren Schullaufbahnen über. Und bei mangelhaften Deutschnoten gibt es ebenfalls jeweils unterschiedliche Übertrittsraten für die miteinander verglichenen Sozialschichten.

Was macht in diesem Fall die Bildungschancen aus - die primären oder die sekundären Herkunftseffekte? Betrachten wir dies für das Jahr 1998. Würde unterstellt werden, die Kinder von Nichtakademikern hätten bei gleichbleibenden Übertrittsraten die gleiche Leistungsverteilung wie Akademikerkinder, dann würden $(61,6 \% \cdot 0,982+30,6 \% \cdot 0,502+7,8 \% \cdot 0,029=) 76$ statt 60 Prozent der Kinder von Nichtakademikern auf die Sekundarschule oder auf das Langzeitgymnasium wechseln. Die Neutralisierung von primären Herkunftseffekten, indem die mittlere Leistung der Kinder von Nichtakademikern verbessert wird, bedeutet zugleich eine deutliche Reduktion sozial ungleicher Bildungschancen. Sie ist deutlicher, als wenn die sekundären Herkunftseffekte neutralisiert werden. In diesem Falle würden $(34,0 \% \cdot 0,993+52,1 \% \cdot 0,657$ $+13,9 \% \cdot 0,118=)$ 69,6 Prozent dieser Kinder auf die höheren Schullaufbahnen gelangen. In der gleichen Grössenordnung (Steigerung von 16 bzw. 7 Prozentpunkten) würden sich die Bildungschancen für die Übergangskohorte 
2009 verbessern. Bei neutralisiertem primärem Herkunftseffekt würden $(54,9 \%$ $\cdot 0,962+37,4 \% \cdot 0,429+7,7 \% \cdot 0,011=) 69$ statt 57 Prozent und bei neutralisiertem sekundärem Herkunftseffekt $(38,3 \% \cdot 0,991+45,9 \% \cdot 0,533+15,8 \%$ $\cdot 0,065=) 64,3$ statt 57 Prozent der sozial benachteiligten Kinder auf die «Sek A» und das Gymnasium wechseln.

Obwohl die Bildungsexpansion, die in der Schweiz im Vergleich zu anderen Ländern zögerlich verlief (Becker \& Zangger, 2013; Buchmann \& Charles, 1993; Buchmann et al., 2007), hat sie zwar Niveaueffekte (d.h. Höherqualifikation in der Bevölkerung), aber keine gravierenden Struktureffekte (d.h. Konstanz der Sozialstruktur beim Bildungsstand) - ausser beim Geschlecht mit sich geführt. Bei einer vergleichbaren Entwicklung im Westen Deutschlands wurde die «enorme Bildungsexpansion in den vergangenen Jahrzehnten (...) im öffentlichen Bewusstsein weithin als Erfolg durchgesetzter Chancengleichheit verstanden (...)» (Friedeburg, 1997, S. 45). Zu Recht wird hierbei darauf hingewiesen, dass diese Deutung der Bildungsexpansion die unterschiedliche Entwicklung relativer Bildungschancen in den einzelnen Bevölkerungsgruppen verkennt. Wie sich die Situation in der Schweiz in den Jahren der Bildungsexpansion seit 1950 entwickelt hat, müsste untersucht werden.

Ebenso wäre es für die Schweiz interessant, der Frage nachzugehen, warum es immer noch - trotz oder wegen der Bildungsexpansion - deutliche Bildungsungleichheiten zwischen den Sozialschichten gibt. Warum gibt es immer mehr Bildungsmöglichkeiten, aber keinen Ausgleich bei den Bildungschancen? Einzelne Antworten wurden bereits geliefert (vgl. Becker, 2010, 2012; Buchmann et al., 2007): Zum einen liegt es an der sozialen Ungleichheit ausserhalb des Bildungssystems, und zum anderen liegt es an der Struktur und den institutionellen Regelungen des Bildungssystems. Zu nennen ist einerseits die hochgradige Stratifizierung und Segmentierung des Bildungssystems: Je stärker ein Bildungssystem stratifiziert ist, je mehr Bildungshürden auf dem Weg zur höheren Bildung überwunden werden müssen, je segmentierter die Bildungswege und je breiter die Bildungsangebote an den einzelnen Übergangsstellen im Bildungssystem sind, desto schwerer wiegen sekundäre Herkunftseffekte bei der Entstehung und Reproduktion sozialer Ungleichheit von Bildungschancen. Andererseits liegt es an den Hürden, die bei den einzelnen Übergangsstellen im Bildungssystem überwunden werden müssen: Je rigider die leistungsbezogenen Sortier- und Selektionsleistungen des Bildungssystems sind, desto grösser sind die Gewichte primärer Herkunftseffekte bei den Übergangsstellen im Bildungssystem.

Wie gesehen, sind die Chancen für Bildungsteilhabe und Erwerb von Bildungszertifikaten auch in der Schweiz ungleich verteilt: Sie lassen sich nicht ausschliesslich durch individuelle Leistungen erklären, sondern sind stark vom sozioökonomischen Hintergrund abhängig. Die Folgen für Individuum und Gesellschaft - von Bildungsarmut bis zur Kumulation leistungsfremder Privilegien - machen in der Schweiz wie in anderen europäischen Ländern die 
Chancenungleichheit im Bildungssystem zu einer bedeutenden sozialpolitischen Frage des 21. Jahrhunderts. Dass Bildung eine der wichtigsten sozialen Fragen darstellt, ist auch für die Schweiz unbestritten. Aber worin liegen die zentralen Ursachen von sozial ungleichen Bildungschancen? Warum werden sozial bedingte Bildungsungleichheiten reproduziert? Weshalb werden soziale Ungleichheiten von Bildungschancen akzeptiert, und wie werden sie legitimiert? Welche Zusammenhänge bestehen zwischen Gerechtigkeitsvorstellungen und gesellschaftlicher Legitimation von Bildungsungleichheit? Was könnte man tun, um soziale Ungleichheit von Bildungschancen - Bildungsarmut als ein Extrem und Kumulation von Privilegien im Bildungssystem - zu reduzieren oder gar zu vermeiden?

Vor dem Hintergrund dieser Fragen wurde der SGBF-Kongress «Bildungsungleichheit und Gerechtigkeit - wissenschaftliche und gesellschaftliche Herausforderungen» vom 2. bis zum 4. Juli 2012 durch das Institut für Erziehungswissenschaft der Universität Bern - im Auftrag der Schweizerischen Gesellschaft für Bildungsforschung (SGBF) sowie in Zusammenarbeit mit der Schweizerischen Gesellschaft für Lehrerinnen und Lehrerbildung (SGL), der Schweizerischen Gesellschaft für Soziologie (SGS), dem Eidgenössischen Hochschulinstitut für Berufsbildung (EHB) sowie der Pädagogischen Hochschule Bern (PHBern) in Bern durchgeführt. Auf dem Kongress wurden Fragen zum Verhältnis von Bildung, sozialer Ungleichheit und Gerechtigkeit behandelt. Diskutiert wurden auch Themen wie die Entstehung von Bildungsungleichheit, Folgen von ungerechter Bildungsteilhabe sowie die Entwicklung und Funktion von Gerechtigkeitskonzepten. Migration, sozialer Herkunft, Geschlecht, Politik und Steuerung, Schulstrukturen und Unterrichtsformen, Curriculum, Lehrerinnenund Lehrerbildung, Ausbildung und Arbeitsmarkt wurden ebenso behandelt wie Prozesse der Inklusion und Exklusion im Bildungssystem. Der Jahreskongress hatte sowohl den wissenschaftlichen Austausch als auch die Diskussion bildungspolitischer, bildungsadministrativer und schulpraktischer Aspekte zum Ziel. Auf dem Jahreskongress der Schweizerischen Gesellschaft für Bildungsforschung (SGBF) 2012 präsentierten über 350 Forscherinnen und Forscher aus zehn Ländern in 30 Symposien und 195 Einzelbeiträgen ihre Forschungsergebnisse zu diesem Themenfeld. Aus dieser Vielzahl der Einzelbeiträge wurden für das vorliegende special issue vier Beiträge ausgewählt.

Im Beitrag mit dem Titel «Herkunftseffekte und Gerechtigkeitserleben beim Übergang von der Primarschule in die Sekundarstufe I» untersuchen Caroline Biewer, Christian Wandeler und Franz Baeriswyl unter dem Gesichtspunkt der sozialen Ungleichheit von Bildungschancen und der sozial gerechten Bildungsteilhabe die Folge des Verfahrens für den Übergang von der Primarschule in die Sekundarstufe I im Kanton Fribourg. Trotz Leistungstest in Deutsch und Mathematik als ein zusätzlichem Selektions- und Allokationskriterium neben Schulnoten, Einschätzungen kognitiver Fähigkeiten durch Lehrpersonen und ihre Zuweisungsempfehlung können anhand der Daten für die Übertritts- 
jahrgänge 2009 und 2010 weiterhin primäre und sekundäre Herkunftseffekte nachgewiesen werden. Wie bereits in vielen früheren Untersuchungen (z.B. Mayer \& Müller, 1976; Hadjar 2008), bestätigt sich auch in dieser Studie, dass die Eltern aus unteren Sozialschichten die soziale Selektivität der Übertritte nicht infrage stellen, während die sozial privilegierten Eltern eher Ungerechtigkeiten des Verfahrens wahrnehmen.

Benita Combet untersucht - ausgehend davon, dass in der Schweiz entgegen meritokratischer Prinzipien der Zusammenhang zwischen sozialer Herkunft und erreichtem Bildungsabschluss besonders ausgeprägt ist - in ihrem Beitrag «Zum Einfluss von primären und sekundären Effekten der sozialen Herkunft beim zweiten schulischen Übergang in der Schweiz. Ein Vergleich unterschiedlicher Dekompositions- und Operationalisierungsmethoden", wie sich die soziale Herkunft die Chance strukturiert, nach der obligatorischen Schulzeit das Gymnasium besuchen zu können. Im Vordergrund steht hierbei, unter Kontrolle relevanter Einflussfaktoren, die Grössen von primären und sekundären Herkunftseffekten bestimmen zu können (vgl. Jackson, 2013). Während beim Übergang in die Sekundarstufe I primäre Herkunftseffekte - Einflüsse der sozialen Herkunft auf die schulische Leistungen - dominieren, kann Benita Combet belegen, dass beim Übergang in das Gymnasium in der Sekundarstufe II sekundäre Herkunftseffekte dominieren; demzufolge entscheiden sich Jugendliche aus höheren Sozialschichten bei gleichen Leistungen eher für eine fortgesetzte Schulbildung als Jugendliche aus den unteren Sozialschichten. Damit steht ihr Befund im Einklang mit einer Vielzahl von Studien aus anderen Ländern (vgl. Becker \& Schuchart, 2010).

Mit der zunehmenden Durchlässigkeit des Bildungssystems in Richtung des Hochschulzugangs sind Hoffnung auf mehr Bildungschancen und -gerechtigkeit verbunden. Jacob Kost untersucht mit Daten der Längsschnittstudie TREE in seinem Beitrag für die Schweiz Schul- und Ausbildungswechsel auf der Sekundarstufe II und welche Rolle sie für den Erwerb der gymnasialen Maturität bzw. der Berufsmaturität und für den Hochschuleintritt spielen. So kann Jacob Kost belegen, dass institutionelle Vorkehrungen für mehr Durchlässigkeit im Bildungssystem über horizontale Wechsel in der Sekundarstufe II die Wahrscheinlichkeit eines Hochschulzugangs eher verringern. Es ist daher - anknüpfend an den vorliegenden Forschungsstand - zu vermuten, dass die Bestrebungen, im berufsbildenden Sektor zusätzliche Möglichkeiten zu schaffen, die Studienberechtigung an Universitäten oder Fachhochschulen zu erwerben, kaum geeignet sind, mehr Bildungsgleichheit herzustellen.

Im abschliessenden Beitrag über «Bildungsungleichheiten beim Hochschulzugang nach Geschlecht und Migrationshintergrund: Befunde aus der Schweiz, Deutschland und Frankreich» untersuchen Dorit Griga und Andreas Hadjar den Übergang in das Hochschulsystem. Im internationalen Vergleich werden hierbei auch Ungleichheiten nach Geschlecht und Migrationshintergrund berücksichtigt. Bei Kontrolle der sozialen Herkunft scheint es für die Schweiz und 
Deutschland zuzutreffen, dass männliche Migranten der zweiten Generation gar im Vorteil gegenüber den Vergleichsgruppen beim Hochschulzugang sind. Benachteiligungen von Migrantinnen konnte für keines der Länder nachgewiesen werden. Im Wesentlichen unterstützt dieser Beitrag die eingangs geschilderten Befunde, dass die Nachteile von Migranten im Bildungssystem auf Benachteiligungen wegen ihrer sozialen Herkunft beruhen (vgl. Becker, 2011).

\section{Bibliographie}

Beck, M., Jäpel, F. \& Becker, R. (2010). Determinanten des Bildungserfolgs von Migranten im Schweizer Bildungssystem. In: Quenzel, G. \& Hurrelmann, K. (Hrsg.), Bildungsverlierer-Neue Ungleichheiten (S. 313-337). Wiesbaden: VS Verlag für Sozialwissenschaften.

Becker, R. (2010). Soziale Ungleichheit im Schweizer Bildungssystem und was man dagegen tun könnte. In: M. Neuenschwander und H.-U. Grunder (Hrsg.), Schulübergang und Selektion - Forschungserträge und Umsetzungsstrategien (S. 91-108). Chur: Rüegger.

Becker, R. (2011). Integration von Migranten durch Bildung und Ausbildung - theoretische Erklärungen und empirische Befunde. In: Becker, R. (Hrsg.), Integration durch Bildung. Bildungserwerb von jungen Migranten in Deutschland (S. 11-36). Wiesbaden: VS Verlag für Sozialwissenschaften.

Becker, R. (2012). Der Übergang ins Hochschulstudium: Prozesse und Mechanismen am Beispiel der deutschen Schweiz. In: Bergman, M., Hupka-Brunner, S., Meyer, T. \& Samuel, R. (Hrsg.), Bildung - Arbeit - Erwachsenwerden. Ein interdisziplinärer Blick auf die Transition im Jugend und jungen Erwachsenenalter (S. 305-331). Wiesbaden: VS Verlag für Sozialwissenschaften.

Becker, R. \& Schuchart, C. (2010). Verringerung sozialer Ungleichheiten von Bildungschancen durch Chancenausgleich? Ergebnisse einer Simulation bildungspolitischer Maßnahmen. in: Rolf Becker und Wolfgang Lauterbach (Hrsg.), Bildung als Privileg (S. 413-436). Wiesbaden: VS Verlag für Sozialwissenschaften (4., aktualisierte Auflage).

Becker, R. \& Zangger, C. (2013). Die Bildungsexpansion in der Schweiz und ihre Folgen. Eine empirische Analyse des Wandels der Bildungsbeteiligung und -ungleichheiten mit den Daten der Schweizer Volkszählungen 1970, 1980, 1990 und 2000. Kölner Zeitschrift für Soziologie und Sozialpsychologie, 65, (im Erscheinen).

Becker, R., Jäpel, F. \& Beck, M. (2013). Geschlechterunterschiede im Bildungserfolg. Eine empirische Analyse für Schulkinder im Deutschschweizer Schulsystem unter besonderer Berücksichtigung ihres Migrationshintergrundes. In: Hadjar, A. \& Hupka-Brunner, S. (Hrsg.), Migrationshintergrund, Geschlecht und Bildungserfolg (S. 77-101). Weinheim: Juventa.

Boudon, R. (1974). Education, Opportunity, and Social Inequality. New York: Wiley.

Breen, R., Luijkx, R. Müller, W. \& Pollak, R. (2009). Nonpersistent Inequality in Educational Attainment: Evidence from Eight European Countries. American Journal of Sociology, 114, $1475-1152$.

Breen, R., Luijkx, R. Müller, W. \& Pollak, R. (2010). Long-term Trends in Educational Inequality in Europe: Class Inequalities and Gender Differences. European Sociological Review, 26, 31-48.

Breen, R., Luijkx, R. Müller, W. \& Pollak, R. (2012). Bildungsdisparitäten nach sozialer Herkunft und Geschlecht im Wandel - Deutschland im internationalen Vergleich. In: Becker, R. \& Solga, H. (Hrsg.), Soziologische Bildungsforschung. Sonderheft 52 der Kölner Zeitschrift für Soziologie und Sozialpsychologie, (S. 346-373). Wiesbaden: Springer + VS Verlag für Sozialwissenschaften. 
Buchmann, M., \& Charles, M. (1993). The Lifelong Shadow: Social Origins and Educational Opportunity in Switzerland. In: Yossi, S. \& Blossfeld, H.-P. (Hrsg.), Persistent Inequality: Changing Educational Attainment in 13 Countries, (S. 177-192). Boulder: Westview Press.

Buchmann, M., Sacchi, S., Lamprecht, M. \& Stamm, H. (2007). Tertiary Education Expansion and Social Inequality in Switzerland. In: Shavit, Y., Arum, R. \& Gomoran, A. (Hrsg.), Stratification in Higher Education, (S. 321-348). Stanford: Stanford University Press.

Ditton, H. (2004). Der Beitrag von Schule und Lehrern zur Reproduktion von Bildungsungleichheit. In: Becker, R. \& Lauterbach, W. (Hrsg.), Bildung als Privileg? (S. 251-279). Wiesbaden: VS Verlag für Sozialwissenschaften (1. Auflage).

Friedeburg, L.v. (1997). Differenz und Integration im Bildungswesen der Moderne. Zeitschrift für Sozialisationsforschung und Entwicklungssoziologei, 17, 43-55.

Hadjar, A. (2008). Meritokratie als Legitimationsprinzip. Die Entwicklung der Akzeptanz sozialer Ungleichheit im Zuge der Bildungsexpansion. Wiesbaden: VS Verlag für Sozialwissenschaften.

Hadjar, A. \& Berger, J. 2010). Dauerhafte Bildungsungleichheiten in Westdeutschland, Ostdeutschland und der Schweiz: Eine Kohortenbetrachtung der Ungleichheitsdimensionen soziale Herkunft und Geschlecht. Zeitschrift für Soziologie, 39, 182-201.

Jackson, M. (2013). Social Background and Educational Transitions in England. In: Jackson, M. (Hrsg.): Determined to Succeed? Performance versus Choice in Educational Attainment (S. 253-279). Stanford, CA: University Press.

Jann, B. \& Combet, B. (2012). Zur Entwicklung der intergenerationalen Mobilität in der Schweiz. Schweizerische Zeitschrift für Soziologi, 38, 177-199.

Mayer, K.U. \& Müller, W. (1976). Soziale Ungleichheit, Prozesse der Statuszuweisung und Legitimitätsglaube. In: Hörning, K.H. (Hrsg.), Soziale Ungleichheit (S. 108-134). Darmstadt: Luchterhand.

Moser U. \& Rhyn, H. (1999). Schulmodelle im Vergleich. Eine Evaluation der Leistungen in zwei Schulmodellen der Sekundarstufe I. Aarau: Sauerländer.

Moser U. \& Rhyn, H. (2000). Lernerfolg in der Primarschule. Aarau: Sauerländer.

Moser, U., Buff, A. Angelone, D. \& Hollenweger, J. (2011). Nach sechs Jahren Primarschule. Deutsch, Mathematik und motivational-emotionales Befinden am Ende der 6. Klasse. Zürich: Bildungsdirektion Kanton Zürich. 
